\title{
Una valoración de la enseñanza superior a partir de los salarios esperados y los salarios sombra de los estudiantes universitarios
}

\author{
César Alonso-Borrego \\ Ricardo Mora \\ Antonio Romero-Medina \\ Universidad Carlos III de Madrid
}

\section{Resumen}

En este trabajo aportamos evidencia sobre la valoración económica de la educación universitaria utilizando las declaraciones de estudiantes universitarios españoles sobre sus expectativas de salario al finalizar sus estudios universitarios y sobre su salario sombra, o salario mínimo permanente a cambio del cuál abandonarían los estudios. Para ello, y dada la naturaleza cualitativa de los salarios declarados, se estiman modelos de respuesta ordenada. Con objeto de controlar la posibilidad de que la calidad de la información difiera en función del curso académico en que se encuentra el estudiante, la estimación se hace por submuestras basadas en el curso. Los salarios esperados predichos por el modelo son muy similares para ambos sexos y, como reflejo de la mejora de la información a lo largo del ciclo académico, se acercan más a los observados para cursos avanzados de la carrera. En cuanto a los salarios sombra, las predicciones basadas en el modelo muestran que son mayores para los últimos años de carrera, cuando el coste de oportunidad de abandonar es más alto. Además, encontramos mayores salarios sombra para las mujeres que para los hombres, a pesar de que los salarios observados son sustancialmente menores en las primeras. Este último resultado está en consonancia con el mayor rendimiento relativo de la educación universitaria en el caso de las mujeres, que se plasma en la mayor propensión de éstas a invertir en dicha educación.

Palabras clave: valoración económica de la educación universitaria, salarios esperados, salarios sombra

Clasificación JEL: I23, J24, J31

\begin{abstract}
In this paper we provide evidence on the economic assessment of university education using Spanish university students' statements about their wage expectations when they finish university and about their shadow wage or permanent minimum wage necessary to induce them to quit university. For that purpose, and given the qualitative nature or the declared wages, ordered response models are estimated. In order to allow that the quality of information differs depending on the student's academic year, the estimation is done by course year based subsamples. The expected wages predicted by the model are very similar for both genders and, as a reflection of the improvement in information during the academic cycle, these wages get closer to the ones observed at the most advanced courses. With respect to shadow wages, predictions based on the model show that they are higher for students in the last years of the degree, when the opportunity cost of quitting is higher. Also, we find greater shadow wages for females than for males, even though the observed wages are substantially lower for women. This last result is in agreement with the higher relative return on university education in case of women, which is reflected by the higher propensity of women to invest in this kind of education
\end{abstract}

Keywords: economic assessment of university education, wage expectations, shadow wages

JEL classification: I23, J24, J31 
Agradecimientos: Agradecemos la financiación de la Comunidad de Madrid a través del proyecto de investigación 06/HSE/0170/2004. Los dos primeros autores agradecen la financiación del Ministerio de Educación a través del proyecto de investigación BEC2006-5710. El tercer autor agradece la financiación del Ministerio de Educación a través del proyecto de investigación SEJ2005-06167.

\section{Introducción}

En la última década del siglo XX, España ha culminado un rápido proceso de expansión educativa (véase San Segundo, 1997), consolidando tasas de escolarización en enseñanza superior algo mayores que la media de la OCDE. No obstante, las comparaciones internacionales sugieren que España mantiene un sistema formativo poco equilibrado en relación con otros países de nuestro entorno. Ello se plasma en el gran peso de los estudios más largos y académicos (licenciaturas), en perjuicio de estudios universitarios más cortos y de estudios superiores no universitarios (véanse San Segundo, 2002; Salas y Martín-Cobos, 2006), así como en el déficit existente en graduados de secundaria (Fina y otros, 2000; Petrongolo y San Segundo, 2002) ${ }^{1}$. La práctica gratuidad de los estudios superiores en España, y el nulo control sobre la asistencia y la compatibilidad de los estudios con otras actividades hacen que la valoración social de dichos estudios sea un problema empírico.

Nuestro trabajo intenta aportar respuestas al problema de valoración económica de la educación universitaria a partir de datos microeconómicos relativos a la valoración económica por parte de los jóvenes universitarios de los estudios que éstos cursan. En particular, explotaremos la información que éstos declaran acerca de sus expectativas de salario al finalizar los estudios, por un lado, y el salario mínimo por el que, con un contrato indefinido, estarían dispuestos a abandonar sus estudios, por otro. La información sobre ambas variables nos permite estudiar dos aspectos del problema de elección de estudios universitarios. Por un lado, podemos analizar la valoración de los estudios universitarios por parte de los jóvenes implicados desde ambas ópticas, condicionando en las características personales y familiares así como en el curso en que se encuentran. Por otro lado, basándonos en las valoraciones individuales predichas por nuestros modelos, podemos comparar los salarios esperados al finalizar los estudios universitarios y los salarios sombra con los salarios efectivos observados para trabajadores con titulación universitaria en el mismo rango de edad.

Dado que los valores de nuestras dos variables de interés están recogidos en intervalos discretos correspondientes a distintos rangos monetarios, el tratamiento em-

\footnotetext{
1 Dentro del conjunto nacional, destaca la situación relativa de Madrid como la región con tasa de participación juvenil más elevada en la educación universitaria. En el caso de Madrid, el desequilibrio en la educación superior a favor de titulaciones universitarias largas es aún más acusado que en el conjunto nacional. Sin embargo, cabe señalar que el sistema universitario madrileño (especialmente sus universidades públicas) tienen características que pueden aconsejar su relativa especialización en enseñanzas largas y de carácter más académico (o general) que profesional.
} 
pírico requiere el uso de sendos modelos de respuesta ordenada que tengan en cuenta que los valores observados de ambas variables dependientes tienen un carácter ordinal. No obstante, el conocimiento de los umbrales que determinan cada intervalo permite, además de predecir las probabilidades asociadas a cada categoría salarial, obtener predicciones de salarios esperados y salarios sombra en términos monetarios, condicionales a las características familiares, personales y académicas.

Los dos modelos econométricos se estiman considerando tres submuestras de estudiantes según el curso en el que se encuentran. En particular, se presentan estimaciones separadas para los estudiantes en los dos primeros años de estudios universitarios, en el tercer año, y en los dos últimos años. Los resultados obtenidos para el modelo econométrico del salario esperado muestran que los diferentes factores explicativos combinan el efecto potencial que éstos tienen sobre el salario con el efecto de la calidad de la información individual en las expectativas salariales. Este último efecto parece dominar para los estudiantes próximos a finalizar sus estudios. Asimismo, el cómputo de los salarios esperados a partir del modelo estimado sugiere que las expectativas salariales están sobreestimadas para los estudiantes de ambos sexos en sus primeros cursos, así como para las mujeres cualesquiera que sea el curso en que se encuentran. En cuanto a la especificación del salario sombra, los resultados son coherentes con un modelo sencillo de inversión en educación universitaria. Por ejemplo, el rendimiento académico afecta positivamente a dicho salario sombra. Un resultado interesante es que en el caso de los estudiantes en últimos cursos de la carrera, el hecho de ser mujer tiene un marcado efecto negativo, mientras que el estar buscando empleo afecta positivamente. Las predicciones de los salarios sombra que se derivan de las estimaciones muestra que el salario sombra más alto se encuentra para estudiantes en sus últimos años de carrera (cuando el coste de oportunidad de abandonar es más alto) y que los salarios sombra son en media mayores o iguales para las mujeres que para los hombres.

El trabajo está organizado de la siguiente manera. La sección 2 formula el marco teórico de referencia, a partir de un modelo sencillo de inversión en capital humano, y define las medidas de valoración de la educación universitaria, salarios esperados y salarios sombra, relevantes para nuestro análisis empírico. La sección 3 describe los datos utilizados, tanto la base de datos principal obtenida a partir de una encuesta para el distrito universitario de Madrid, como una base de datos complementaria que nos permite obtener información representativa sobre salarios medios observados por sexo, grupos de edad y nivel de estudios, que utilizaremos para poner en contexto los resultados de nuestro análisis. El modelo econométrico y los resultados empíricos se detallan en las secciones 4 y 5 , respectivamente. Finalmente, la sección 6 concluye resumiendo los principales resultados del trabajo, enumera algunas de las limitaciones del análisis y propone algunas posibilidades de investigación futura. 


\section{Marco teórico}

Con objeto de utilizar un modelo sencillo de inversión en educación universitaria que se adapte a las necesidades de nuestro análisis empírico, hemos tomado el modelo de capital humano presentado en Trostel (2004) como referencia, dado que contiene las peculiaridades relevantes a nuestro análisis empírico, por un lado y es sencillo de tratar, por otro. Típicamente, se supone que el capital humano aumenta linealmente la productividad del trabajo y por lo tanto el salario, $W^{*}$. En otros trabajos como Blinder y Weiss (1976) y Rosen (1976) se propone una definición alternativa, pero esencialmente equivalente, por la que la tecnología de producción del capital humano es lineal aunque la productividad del mismo presenta una estructura no lineal.

$$
W^{*}=r H
$$

donde $r$ representa el coste de uso del capital humano, $H$. Suponemos que la acumulación de capital humano $H$ viene determinada de acuerdo con una función de producción del tipo

$$
d H_{t} / d t=\varphi x_{t}^{a} y_{t}^{y} H_{t}^{\delta}
$$

donde para un individuo dado en un momento del tiempo $t, x$ es el tiempo invertido en capital humano e $y$ representa aquellos bienes invertidos en producir capital humano como los servicios de profesores, el capital físico etc.; el parámetro $\varphi$ representa la productividad o capacidad del individuo. Finalmente $\alpha, \gamma, \delta$ son las elasticidades asociadas a cada una de las respectivas variables. Dado que la depreciación a la que pueda estar sujeta el capital humano no afecta a nuestro análisis, no se tiene en cuenta en este contexto. Para que el modelo tenga solución interior, debe darse la condición de que $\alpha+\gamma<1$.

Siguiendo a Haley (1976), las condiciones de primer orden para la producción óptima se pueden utilizar para sustituir y en la función de producción. En tal caso, la ecuación anterior se convierte en

$$
d H_{t} / d t=\Phi x_{t}^{\alpha+\gamma} H_{t}^{\delta+\gamma}
$$

donde

$$
\Phi \equiv \varphi(y r / \alpha p)^{\gamma}
$$

y $p$ es el precio de $y$.

La información que se utiliza en la medición del nivel de producción del capital humano son los años de educación. Nos centramos por lo tanto en la formación de capital humano a través de la educación. Si suponemos que cada año de educación tiene el mismo impacto en la generación de capital humano a lo largo del tiempo, la 
ecuación puede simplificarse aún más, dado que $x$ se mantiene constante. Si hacemos $x$ igual a la unidad, sin pérdida de generalidad, se obtiene una función de producción de capital humano del tipo

$$
d H_{t} / d t=\Phi H_{t}^{\sigma}
$$

para $0<t<S$, donde $\sigma=\delta+\gamma$, es decir, la elasticidad de los inputs puede acumularse, y $S$ representa los años totales de educación. La ecuación asociada a la función de producción de capital humano es una ecuación de Bernoulli con coeficientes constantes. La solución a esta ecuación al final de $\operatorname{los} S$ años de educación es:

$$
H_{S}=\left\{\begin{array}{cc}
H_{0} e^{\Phi S} & \text { si } \sigma=1 \\
\left(H_{0}^{1-\sigma}+(1-\sigma) \Phi S\right)^{1 /(1-\sigma)} & \text { si } \sigma \neq 1
\end{array}\right\}
$$

donde $H_{0}$ es el stock de capital humano previo a la escolarización. Idealmente, podría contrastarse si el valor de la elasticidad $\Phi$ es igual a la unidad. Alternativamente, si los datos no lo permiten, puede imponerse la restricción de que su valor es igual a 1. En ese caso, sustituyendo la solución anterior en la relación entre salario y capital humano, $W^{*}=r H$ y tomando logaritmos para linealizar la expresión resultante, se obtiene una expresión que muestra que el rendimiento del capital humano puede inferirse de la relación empírica entre el logaritmo del salario y los años de escolarización:

$$
\ln W^{*}=\ln r+\ln H_{0}+\Phi S .
$$

Dado que el capital humano previo a la escolarización es una función de factores observables recogidos en el vector $Z$ como los que caracterizan el entorno sociodemográfico del individuo e inobservables $v$, parametrizaremos $H_{0}$ como

$$
H_{0}=\exp \left(\theta_{0}+\theta_{1}{ }^{\prime} Z+v\right)
$$

de manera que, teniendo en cuenta que $r$ es constante, podemos escribir la especificación como

$$
\ln W^{*}=\alpha+\Phi S+\theta_{1}{ }^{\prime} Z+v
$$

\subsection{Salarios esperados}

La ecuación anterior establece una sencilla relación lineal entre los salarios observados y los niveles de escolarización de cada asalariado que, bajo determinados supuestos, permite obtener sin dificultad las expectativas medias de salarios futuros de los estudiantes universitarios. 
Suponiendo, sin pérdida de generalidad, que en promedio el término $v$ se iguala a cero, el salario esperado dados $r, S, Z$ es $\alpha+\Phi S+\theta_{l}{ }^{\prime} Z$. Por otro lado, para un estudiante que se encuentre en el año $k$ de sus estudios universitarios, su expectativa de salario al finalizar sus estudios dependerá del conjunto de información que dicho estudiante utiliza para calcular dicha expectativa. En concreto:

$$
\mathrm{E}_{\mathrm{k}}\left(\mathrm{In} W^{*}\right)=\alpha_{k}^{e}+\Phi_{k}^{e} S+\theta_{1 k}^{e} Z+E_{k}(v)
$$

donde $E_{k}(\cdot)$ representa la esperanza matemática condicional al conjunto de información disponible para el estudiante en el curso $k$, y $\alpha_{k}^{e}, \Phi_{k}^{e}, \theta_{1 k}^{e}$ representan las esperanzas de los rendimientos en la ecuación de salarios condicionales al mismo conjunto de información. Suponiendo que $E_{k}(v)$ es igual a cero, entonces el salario esperado medio toma el valor $\alpha_{k}^{e}+\Phi_{k}^{e} S+\theta_{1 k}^{e} Z$.

De todo lo anterior se deduce que las diferencias entre salarios medios y expectativas medias de salarios provienen de las diferencias entre las expectativas de los rendimientos en la ecuación de salarios y los valores efectivos de éstos:

$$
\left[E_{k}\left(\ln W^{*}\right)-E\left(\ln W^{*}\right)\right]=\left(\alpha_{k}^{e}-\alpha\right)+\left(\Phi_{k}^{e}-\Phi\right) S+\left(\theta_{1 k}^{e}-\theta_{1}\right)^{\prime} Z
$$

Obsérvese que estas diferencias entre los salarios medios efectivos y sus expectativas medias dependen del nivel de información de los agentes. Es razonable suponer que las expectativas del estudiante dependerán del curso académico en el que se encuentre por dos motivos. En primer lugar, el curso académico refleja en parte el nivel de conocimiento que el estudiante tiene sobre la disciplina en la que se está formando. En segundo lugar, también refleja el número de años de educación que todavía requiere antes de obtener un salario, es decir, el horizonte de predicción. Por ello, a la hora de evaluar las expectativas sobre el rendimiento de la educación, el error de predicción de un estudiante al inicio de sus estudios universitarios será superior que el error de predicción de ese mismo estudiante cuando está finalizando dichos estudios. El modelo, por tanto, establece una relación decreciente entre el curso académico y las disparidades entre los salarios medios y las expectativas de salarios.

\subsection{Salarios sombra}

Es importante distinguir dentro de nuestro modelo entre los conceptos de salario esperado y salario sombra. Definimos salario sombra como el salario mínimo, en términos reales, por el cual el individuo estaría dispuesto a abandonar sus estudios e incorporarse al mercado de trabajo percibiendo dicho salario a lo largo de toda su vida laboral. Por tanto, consideramos que dicho salario permanecerá constante en térmi- 
nos reales a lo largo del tiempo ${ }^{2}$. Además, aquellos estudiantes que abandonan sus estudios dejan de soportar los costes directos e indirectos de la adquisición de capital humano, es decir los costes asociados a la adquisición de formación, por un lado, y el coste de oportunidad del tiempo, por otro.

Concentrándonos en salarios reales, si fijamos una edad de retirada del mercado laboral en el periodo $n$ con idénticos beneficios y consideramos para simplificar que el tipo de interés real es igual a cero, podemos definir formalmente nuestro salario sombra $w^{s}$ para alguien que abandona sus estudios $s$ años después de iniciarlos:

$$
w^{s}=\frac{\sum_{t=s}^{n} W_{t}}{(n-s)}
$$

Nuestro modelo establece dos predicciones relativas a los salarios sombra. En primer lugar, si los salarios en términos reales aumentan con la experiencia laboral de los individuos a una tasa suficiente para compensar los años adicionales de escolarización, los salarios esperados al finalizar los estudios deben ser inferiores a los salarios sombra. En segundo lugar, en la medida en que los rendimientos de la educación universitaria sean positivos, los salarios sombra deben aumentar con los años de estudio.

\section{Los datos}

\subsection{Los jóvenes ante la Universidad}

En 1990, el Centro de Investigaciones Sociológicas, en colaboración con el Ministerio de Educación, llevó a cabo una encuesta de ámbito nacional tanto a estudiantes preuniversitarios como estudiantes universitarios con el objetivo de proporcionar una imagen precisa de las actitudes y las opiniones de los jóvenes estudiantes españoles ante el sistema educativo, y en especial ante el sistema de acceso a la universidad y a las reformas que se habían producido recientemente. La encuesta y el informe posterior recibieron el nombre de «Los jóvenes ante el sistema educativo» (CIS, 1991). Con el objeto de actualizar en el ámbito universitario madrileño la información recogida en dicha encuesta, un equipo investigador de la Universidad Carlos III de Madrid, con la financiación de la Comunidad de Madrid, ha diseñado y llevado a cabo desde el año 2000 una encuesta cuyo objetivo fundamental es recoger una imagen precisa año a año de las actitudes de los jóvenes estudiantes madrileños ante el proceso de admisión en el sistema público de universidades.

2 Como se comentará en la sección siguiente, del modo en que se pregunta a los encuestados sobre dicho salario sombra, se supone que dicho salario permanecerá constante en términos reales a lo largo del tiempo. 
Esta encuesta, denominada Los Jóvenes ante la Universidad, es pertinente para el presente trabajo, ya que, a diferencia de la encuesta original del CIS, recaba información sobre la valoración de los estudios universitarios en términos del coste de oportunidad para cada estudiante. En concreto, además de recoger información sobre las características personales del estudiante y de sus padres, la encuesta proporciona información sobre las expectativas de ingresos de los estudiantes una vez finalicen sus estudios superiores, así como sus salarios sombra.

La población objetivo la forman los estudiantes universitarios en universidades públicas de la Comunidad de Madrid durante los cursos académicos 2003/2004 y 2004/2005. Dentro de la población se realiza la encuesta sólo a aquellos estudiantes que se incorporaron a la Universidad a partir de COU/LOGSE o que se encuentran cursando las distintas ramas de las enseñanzas medias.

La encuesta proporciona información crucial en las cuestiones que abordamos en este trabajo. En particular, dos preguntas hacen referencia a los salarios esperados al finalizar los estudios universitarios y a los salarios sombra. Respecto a los salarios esperados, a cada estudiante se le pregunta cuál piensa que será su salario mensual al finalizar los estudios. Concretamente, la pregunta es: «¿Qué salario mensual esperas obtener al finalizar los estudios?». Las respuestas de los alumnos están censuradas a seis posibles categorías, además de no contestar, o declarar que no saben contestar. Las categorías son: 450 euros, 901 euros, 1.803 euros, 3.606 euros, 5.409 euros, y más de 5.409 euros. Respecto a los salarios sombra, a los estudiantes se les pregunta « $i \mathrm{~A}$ partir de qué salario mensual aceptarías dejar tus estudios firmando un contrato de trabajo permanente?», ofreciéndoles las mismas categorías que en la pregunta anterior.

En la Tabla 1 se presentan las frecuencias marginales de las respuestas de los estudiantes a las dos preguntas sobre salarios esperados y salarios sombra. Mientras que los salarios esperados presentan un marcado perfil unimodal, por el que un 63 por ciento de los estudiantes respondieron un salario esperado de 1.803 euros, los salarios sombra se distribuyen de forma mucho más uniforme por encima de la categoría mínima de 450 euros. No obstante, el coeficiente de correlación ordinal de Kendall es 0,21, con un $p$-valor inferior a 0,0001 , lo que confirma una fuerte correlación de rango positiva entre las dos variables.

Además de estas dos variables, la encuesta recoge información sobre los encuestados tanto de su situación académica como de su entorno socioeconómico. En lo que respecta a la situación académica, se dispone de información sobre el curso académico dentro del plan de estudios de la titulación en que se encuentra matriculado el estudiante, así como si es repetidor y sobre la senda (ciencias, letras o mixta) que el individuo cursó en sus estudios preuniversitarios. Asimismo, se dispone de información sobre su rendimiento académico anterior a su acceso a la Universidad, a través de su nota de selectividad, así como si el estudiante busca trabajo y si disfruta de beca de estudios. Por otro lado, la base de datos contiene información detallada sobre el nivel de estudios tanto del padre como de la madre.

En la Tabla 2 se presenta un cuadro resumen de varios estadísticos descriptivos concernientes a las variables de control utilizadas en el presente trabajo. 
TABLA 1

SALARIOS ESPERADOS MENSUALES Y SALARIOS SOMBRA EN LOS ESTUDIANTES UNIVERSITARIOS DE LA COMUNIDAD DE MADRID. DISTRIBUCIÓN DE FRECUENCIAS (\%)

\begin{tabular}{|l|c|c|}
\hline & $\begin{array}{c}\text { Salarios } \\
\text { esperados }\end{array}$ & $\begin{array}{r}\text { Salarios } \\
\text { sombra }\end{array}$ \\
\hline 450 euros & 0,78 & 1,41 \\
901 euros & 12,51 & 22,91 \\
1803 euros & 62,75 & 19,13 \\
3.606 euros & 16,35 & 25,67 \\
5.409 euros & 2,81 & 11,75 \\
Más de 5.409 euros & 4,79 & 19,13 \\
Número de observaciones & 1.670 & 1.558 \\
\hline
\end{tabular}

Fuente: Los Jóvenes ante la Universidad, 2004 y 2005.

TABLA 2

ESTADÍSTICOS DESCRIPTIVOS DE VARIABLES DE CONTROL

\begin{tabular}{|l|r|c|c|c|}
\hline \multicolumn{1}{|c|}{ Variable } & Media & $\begin{array}{c}\text { Desviación } \\
\text { Típica }\end{array}$ & Mínimo & Máximo \\
\hline Mujer & 0,55 & 0,50 & 0 & 1 \\
Curso & 2,50 & 1,18 & 1 & 5 \\
Repetidor & 0,33 & 0,47 & 0 & 1 \\
Ciencias & 0,51 & 0,50 & 0 & 1 \\
Selectividad & 67,59 & 9,03 & 50 & 99 \\
Busca & 0,36 & 0,48 & 0 & 1 \\
Beca & 0,17 & 0,38 & 0 & 1 \\
\hline
\end{tabular}

Fuente: Los Jóvenes ante la Universidad, 2004 y 2005.

Salvo Selectividad y Curso, todas las variables presentes en la Tabla 2 son variables dicotómicas. Mujer toma el valor 1 si el estudiante encuestado es mujer y cero en caso contrario. Repetidor indica si el encuestado ha repetido al menos un curso. Ciencias indica si el estudiante cursó las sendas de Ciencias de la Naturaleza y Salud ó Tecnología en los estudios LOGSE. Busca refleja si el estudiante busca trabajo mientras que Beca señala si disfruta de una beca de estudios. Aproximadamente la mitad de los encuestados son mujeres; un tercio ha repetido al menos un curso; la mitad provienen de una senda de ciencias; un tercio busca trabajo, y menos de un 
quinto disfruta de una beca de estudios. Finalmente, la variable Curso nos indica el curso en el que se encuentra el estudiante. La variable Selectividad indica la nota de selectividad obtenida por cada encuestado, con un rango comprendido entre un mínimo de 50 puntos y un máximo de 99 sobre 100 .

Como cabe esperar, el porcentaje de alumnos en cursos superiores es ligeramente inferior al de alumnos en los primeros años de los estudios. En concreto, mientras que el 50 por ciento de la muestra corresponde a alumnos en los dos primeros años de los estudios universitarios, solo el 18 por ciento de los encuestados declaran encontrarse en cuarto o quinto de sus estudios universitarios.

Como hemos indicado anteriormente, la encuesta Los Jóvenes ante la Universidad también proporciona información sobre los niveles de estudios del padre y de la madre. Se consideran inicialmente 8 categorías de estudios diferentes: no sabe leer, sabe leer, estudios primarios completos, estudios de formación profesional, bachiller elemental, bachiller superior, estudios de grado medio y estudios universitarios. Los niveles de estudios del padre y de la madre están estrechamente correlacionados. El coeficiente MCO de la proyección lineal del nivel de estudios de la madre sobre el nivel de estudios del padre presenta un estadístico $t$ para el coeficiente del nivel de estudios del padre de 28,72. La correlación ordinal entre ambas variables es positiva y muy significativa (el coeficiente de correlación ordinal de Kendall es 0,46 y su $p$-valor es inferior a 0,0001 ). De todas las categorías sobre el nivel de estudios del padre, la más frecuente es la de estudios universitarios, alcanzando un 24 por ciento de la muestra total. Las restantes categorías fluctúan entre el 10 y el 18 por ciento, a excepción de las dos inferiores, que conjuntamente suponen solamente un 15 por ciento de la muestra. Es decir, en relación con la población española, nuestra muestra está sesgada en el sentido de que los individuos encuestados tienen padres cuyo nivel de estudios medio es ligeramente superior al nivel de estudios medio de los españoles con hijos en edad de cursar estudios universitarios. La misma conclusión se obtiene si se analiza la representatividad de la muestra en relación al nivel de estudios de la madre. Este sesgo es previsible y compatible con la fuerte inercia intergeneracional observada en los niveles de estudios dentro de una misma familia (véase, por ejemplo, la Encuesta de Conciencia, Biografía y Estructura de Clase de 1991). Nuestra especificación preferida incluirá simplemente una variable binaria que tomará valor uno para los estudiantes con padre con título universitario, Padre universitario.

Por último, a los estudiantes se les preguntó por el nivel de ingresos del hogar. Aunque las respuestas admisibles estaban restringidas a seis categorías para facilitar la contestación por parte de los estudiantes, un porcentaje elevado de éstos declararon no saber cuáles eran los ingresos medios anuales de su hogar. Por esta razón, además de que los ingresos declarados están altamente correlacionados con las niveles de educación del padre y de la madre, hemos optado por explotar únicamente dentro del análisis empírico la información de si el estudiante declara un nivel de ingresos del hogar, o por el contrario, decide no contestar a tal pregunta. La variable binaria que 
representa tal información, Declara, tiene una media de 37,22, indicando que más de dos tercios de los estudiantes de la muestra no conocen o no revelan el nivel de ingresos del hogar.

\subsection{La Encuesta de Estructura Salarial}

La Encuesta de Estructura Salarial es una base de datos elaborada por el Instituto Nacional de Estadística (en adelante, INE) con el fin de estudiar la estructura y distribución de los salarios en España. Esta encuesta investiga la distribución de los salarios en función de una gran variedad de variables como son la edad, el sexo, el nivel de estudios y la comunidad autónoma de residencia. Aunque se realizó por primera vez en 1995, en esta sección haremos uso de los datos presentados en su segunda edición, en 2002, para enmarcar la evidencia sobre salarios esperados y salarios sombra obtenidos en la encuesta Los Jóvenes ante la Universidad.

En la Tabla 3 presentamos la ganancia media mensual en euros por trabajador con edad comprendida entre los 20 y 29 años. La información de esta tabla es de carácter público y fácilmente accesible en las publicaciones del INE y en su página de Internet. En este sentido, parece razonable que los estudiantes universitarios formen

TABLA 3

GANANCIA MEDIA MENSUAL POR TRABAJADOR DE 20 A 29 AÑOS (EUROS)

\begin{tabular}{|c|c|c|c|c|}
\hline & $\begin{array}{l}\text { Educación } \\
\text { secundaria }\end{array}$ & $\begin{array}{l}\text { Formación } \\
\text { profesional }\end{array}$ & $\begin{array}{c}\text { Diplomados } \\
\text { universitarios }\end{array}$ & $\begin{array}{c}\text { Educación } \\
\text { superior }\end{array}$ \\
\hline Total Nacional & & & 177451 & \\
\hline $\begin{array}{l}\text { Ambos sexos } \\
\text { Varones }\end{array}$ & $\begin{array}{l}1.356,70 \\
1.538,09\end{array}$ & $\begin{array}{l}1.359,32 \\
1.554,27\end{array}$ & $\begin{array}{l}1.774,51 \\
1.999,15\end{array}$ & $\begin{array}{l}1.995,50 \\
2.177 .94\end{array}$ \\
\hline Mujeres & $1.191,71$ & $1.146,98$ & $1.614,58$ & $1.843,18$ \\
\hline \multicolumn{5}{|c|}{ Comunidad de Madrid } \\
\hline Ambos sexos & $1.416,62$ & $1.388,95$ & $1.960,50$ & $2.226,32$ \\
\hline Varones & $1.617,09$ & $1.578,36$ & $2.190,94$ & $2.504,85$ \\
\hline Mujeres & $1.243,43$ & $1.223,27$ & $1.769,15$ & $2.001,99$ \\
\hline \multicolumn{5}{|c|}{ Desviación porcentual de las ganancias medias en la Comunidad de Madrid } \\
\hline Ambos sexos & 4,42 & 2,18 & 10,48 & 11,57 \\
\hline Varones & 5,14 & 1,55 & 9,59 & 15,01 \\
\hline Mujeres & 4,34 & 6,65 & 9,57 & 8,62 \\
\hline
\end{tabular}


sus expectativas personales teniendo en cuenta esta información. No obstante, es importante enfatizar que los salarios medios observados en esta base de datos son representativos de la población que voluntariamente quiere trabajar al salario ofrecido en el mercado de trabajo español. Por tanto, pueden estar sujetos a dos sesgos de selección. En primer lugar, el sesgo originado por la decisión de trabajar, que afecta principalmente a los trabajadores de sexo femenino, ya que incluso en este tramo de edad de 20 a 29 años presentan una tasa de participación inferior a la de los varones. En segundo lugar, el sesgo originado por la decisión de ser asalariado. En la encuesta realizada a los estudiantes universitarios, no es posible controlar por esta selección de la muestra, ya que ambas decisiones se producen después de terminar los estudios universitarios y pueden estar condicionadas por eventos que tienen lugar tras la realización de la encuesta.

En cualquier caso, tomaremos los datos de la Tabla 3 como los datos de referencia para evaluar las expectativas y los salarios sombra de los estudiantes universitarios de la Comunidad de Madrid.

Del análisis de la Tabla 3, cabe destacar tres aspectos. En primer lugar, existe una relación positiva entre nivel de estudios y ganancias. En segundo lugar, los hombres obtienen en promedio ganancias superiores a las mujeres para un mismo nivel de estudios. Finalmente, los asalariados de la Comunidad de Madrid disfrutan en promedio de salarios superiores a la media nacional. A continuación, comentamos estos tres rasgos detalladamente.

Como cabría esperar del marco teórico expuesto en la sección 2, el nivel de estudios está positivamente correlacionado con las ganancias medias observadas de los trabajadores, con independencia de su localización y género. A mayor nivel de estudios, mayores son las ganancias medias.

Por supuesto, parte de este diferencial puede ser debido a que los individuos con mayor capacidad para los estudios y la vida profesional tienden a invertir más en su capital humano, generando un diferencial no aleatorio entre el nivel de estudios y las ganancias observadas en la base de datos. En el presente trabajo y por razones de brevedad no vamos a controlar por este sesgo de selección, sino que supondremos que los estudiantes encuestados en las universidades tienen niveles de habilidad inobservables parecidos a los de los trabajadores con estudios superiores de la Encuesta de Estructura Salarial. Por ello, independientemente de la causa fundamental por la que se observa, nos limitaremos a estudiar si los estudiantes universitarios esperan niveles de salarios con una brecha educacional superior o inferior a la observada para los trabajadores con estudios superiores.

La Tabla 4 presenta los rendimientos a la educación observados en la Comunidad de Madrid. Puede verse que los diferenciales de ganancias medias entre los trabajadores de estudios superiores y los trabajadores con niveles inferiores no universitarios se encuentran situados alrededor del 60 por ciento. Este diferencial, sin embargo, no refleja realmente las diferencias por año de estudios de ganancias medias a lo largo del ciclo vital por dos razones. En primer lugar, los trabajadores con niveles de estudios superiores entran en media en el mercado laboral después de los 
TABLA 4

RENDIMIENTOS MEDIOS DE LA EDUCACIÓN UNIVERSITARIA EN LA COMUNIDAD DE MADRID

\begin{tabular}{|c|c|c|c|}
\hline \multicolumn{4}{|c|}{$\begin{array}{c}\text { Variación porcentual de la ganancia media mensual de licenciados, } \\
\text { ingenieros superiores y doctores }\end{array}$} \\
\hline & $\begin{array}{l}\text { Educación } \\
\text { secundaria }\end{array}$ & $\begin{array}{l}\text { Formación } \\
\text { profesional }\end{array}$ & $\begin{array}{l}\text { Diplomados } \\
\text { universitarios }\end{array}$ \\
\hline Varones & 54,90 & 58,70 & 14,33 \\
\hline Mujeres & 61,01 & 63,66 & 13,16 \\
\hline \multicolumn{4}{|c|}{$\begin{array}{l}\text { Variación porcentual del rendimiento medio descontado sobre el ciclo vital de un año } \\
\qquad \text { de educación superior adicional }\end{array}$} \\
\hline & $\begin{array}{l}\text { Educación } \\
\text { secundaria }\end{array}$ & $\begin{array}{l}\text { Formación } \\
\text { profesional }\end{array}$ & $\begin{array}{l}\text { Diplomados } \\
\text { universitarios }\end{array}$ \\
\hline Varones & 7,32 & 10,77 & 4,75 \\
\hline Mujeres & 4,84 & 9,64 & 4,84 \\
\hline
\end{tabular}

Fuente: INE y elaboración propia.

trabajadores con niveles inferiores de estudios ya que la mayoría de ellos no trabajan mientras están estudiando. Además, el número de años que requiere cada titulación no es constante, existiendo titulaciones, tales como las diplomaturas, donde el número de años es inferior a cuatro. Como se observa en el panel superior de la Tabla 4, el diferencial total entre las ganancias de los diplomados y los licenciados es mucho menor que el diferencial entre los licenciados y los trabajadores con educación secundaria o formación profesional en grado medio. En el panel inferior de la Tabla 4 se corrigen los rendimientos a la educación teniendo en cuenta tanto los años sacrificados por los universitarios para su inversión en capital humano, como el número de años mínimo exigidos para completar cada nivel de estudios. Una vez realizadas estas correcciones se observa que los rendimientos a la educación de licenciados hombres y mujeres son, respectivamente, 7,3 y 4,8 por ciento para los estudiantes de secundaria y 10,8 y 9,6 por ciento respecto a los estudiantes de formación profesional.

En cuanto al segundo aspecto observado en la Encuesta acerca del diferencial en las ganancias medias según el sexo del trabajador, dicho diferencial se produce para todos los niveles de estudios, si bien se estrecha a medida que el nivel de estudios aumenta. En particular, el diferencial se sitúa entre el 15 y el 20 por ciento a favor de 
los varones con niveles de estudios superiores. Existen numerosas causas potenciales de la existencia de esta brecha salarial por sexo para un grupo de edad tan bajo aun controlando por niveles de estudios. Entre ellas, cabe mencionar la existencia de discriminación salarial, la posibilidad de que las mujeres trabajadoras acumulen en promedio menor capital humano específico de las empresas (dada su mayor probabilidad de que se produzcan discontinuidades en su vida laboral) y la segregación ocupacional. En este último caso, es posible que parte de las mujeres trabajadoras se enfrenten a restricciones que les llevan a elegir ocupaciones con bajos niveles salariales que les proporcionan compensaciones no salariales, tales como mayor flexibilidad de horarios. En cualquier caso, y de forma análoga al comentario relativo a los niveles de educación, conviene aclarar que nuestro estudio se centra en indagar si los estudiantes declaran expectativas cercanas a las que un observador de la Encuesta de Estructura Salarial puede obtener.

Respecto al mayor salario relativo observado entre los trabajadores dentro de la Comunidad de Madrid frente a todos los trabajadores del ámbito nacional, dicho diferencial se observa para todos los niveles de estudios pero es creciente con éstos. Además, no muestra una pauta clara con respecto al sexo del trabajador. Entre las causas de este diferencial se hallan tanto el mayor coste de la vida en la Comunidad de Madrid como una mayor productividad del trabajo. Los estudiantes universitarios en la Comunidad de Madrid provienen de varias regiones de España. Si bien la mayoría probablemente se quedarán en Madrid tras sus estudios, un porcentaje significativo aceptará trabajos en otros lugares donde el nivel de salarios es inferior. Es por ello por lo que conviene hacer la comparación de los salarios esperados frente a los salarios de trabajadores con nivel superior de estudios considerando dos escenarios. En el primer escenario, todos los estudiantes se quedarían a trabajar en la Comunidad de Madrid. Bajo este escenario, los salarios medios de referencia relevantes son los salarios medios de los trabajadores de la Comunidad de Madrid. En un segundo escenario, probablemente menos realista, los estudiantes aceptarían trabajos en las diferentes regiones de España en proporción al tamaño relativo de cada región respecto al total nacional. Bajo este escenario, los salarios de referencia relevantes serían los salarios medios observados en España.

\section{Un modelo econométrico sobre la valoración de los estudios universitarios}

Para especificar el modelo empírico, partimos de la ecuación obtenida en la sección 2 para el logaritmo del salario, para un individuo dado

$$
\ln W_{i}^{*}=\alpha+\Phi S_{i}+\theta_{1}{ }^{\prime} Z_{1}+v_{i} \quad(i=1, \ldots, n)
$$

Dicha ecuación caracteriza los determinantes del salario que recibe cada individuo en función de su nivel de educación y de su entorno sociodemográfico. 
Sin embargo, a diferencia de la información objetiva proporcionada por los salarios observados para individuos que trabajan, en nuestro caso nos centramos en la información sobre la valoración subjetiva que los propios estudiantes tienen acerca de la enseñanza superior. Dicha valoración se plasma tanto en los salarios que esperan obtener los individuos que están realizando los estudios universitarios como en los salarios sombra de éstos, es decir, el salario en un puesto de trabajo con contrato indefinido a cambio del cual estarían dispuestos a renunciar a sus estudios universitarios. Esta información permite analizar la valoración de la educación superior por parte de los jóvenes desde la doble óptica de los ingresos esperados y del salario sombra.

Tal y como se han descrito las características de nuestra base de datos en la sección anterior, disponemos de información sobre estudiantes matriculados en distintos cursos, y en consecuencia con diferencias tanto en sus niveles de educación como en la calidad y cantidad de información de que disponen para el cómputo de su salario esperado y de su salario sombra. Por ello, el curso en que se encuentran puede afectar, manteniéndose otros factores constantes, a la valoración subjetiva de los estudios universitarios. El hecho de que existan diferencias en el horizonte de terminación de los estudios abre la posibilidad de que existan diferencias en cómo los individuos generan dichas valoraciones subjetivas. En particular, los efectos de los factores explicativos que vamos a considerar pueden diferir en función del curso académico en que se encuentra cada individuo. En consecuencia, estimaremos el modelo para tres distintas submuestras basadas en el momento en que se hallan cursando sus estudios: primer y segundo curso, tercer curso, cuarto y quinto curso. En tal caso, el nivel de estudios $S_{i}$ es constante en cada submuestra, de manera que $\Phi S_{i}$ formará parte del término constante del modelo.

Además de las variables que caracterizan el entorno sociodemográfico (asociado al capital humano previo a la escolarización), es preciso tener en cuenta características individuales que determinan la calidad de dicha valoración, como por ejemplo el historial académico y la especialidad cursada en secundaria, además de otras características individuales como el sexo. Estas características ya han sido descritas en la sección anterior que describía la base de datos. Por tanto, ampliaremos el vector de características que afectan a la valoración $\ln W_{i}^{*}$, resumidas por el vector de variables $X_{i}$. Asimismo, tendremos en cuenta el efecto de factores inobservables adicionales (además de los factores inobservables que afectan al capital humano previo a la escolarización) que pueden afectar a la valoración, expresados en el término de error del modelo $u_{i}$. Podemos escribir por tanto la especificación empírica como

$$
\ln W_{i}^{*}=\beta^{\prime} X_{i}+u_{i} \quad(i=1, \ldots, n)
$$

Si observamos $W_{i}^{*}$, el estimador del vector de parámetros $\beta$ (que incluye término constante) puede obtenerse aplicando MCO. En la práctica, tal y como se ha des- 
crito en la sección anterior, $W_{i}^{*}$ se observa de forma discreta en seis rangos de valores $^{3}$. Formalmente, la variable observable $W_{i}$ puede definirse como

$$
W_{i}=j \text { si } \mu_{j-1}<W_{i}^{*}<\mu_{j}(j=1, \ldots, 6)
$$

Podemos asimismo definir indicadores binarios asociados a cada categoría como

$$
d_{i j}=\mathbf{1}\left(W_{i}=j\right)=\mathbf{1}\left(\mu_{j-1}<W_{i}^{*}<\mu_{j}\right) .
$$

Este problema de observabilidad imposibilita estimar el vector de parámetros de interés aplicando MCO. Para tratar este problema, consideramos un modelo de respuesta ordenada, en el que explotamos la información discreta acerca de los rangos salariales declarados por los individuos.

$$
\mu_{0}<\mu_{1}<\cdots<\mu_{6}
$$

Como $W_{i}^{*}$ sólo se observa ordinalmente, utilizamos la normalización $\operatorname{Var}\left(u_{i} \mid X_{i}\right)=$ 1. Nuestro problema de estimación incluye los parámetros de interés, así como los parámetros $\mu_{j}$ que definen los umbrales de las seis categorías. Podemos escribir por tanto la probabilidad de escoger la categoría $j$-ésima como

$$
\begin{aligned}
\operatorname{Pr}\left(W_{1}\right. & \left.=j \mid X_{i}\right)=\operatorname{Pr}\left(d_{i j}=1 \mid X_{i}\right)=\operatorname{Pr}\left(\mu_{j-1}<W_{i}^{*}<\mu_{j} \mid X_{i}\right) \\
& =\operatorname{Pr}\left(\ln \mu_{j-1}-\beta^{\prime} X_{i}<\ln W^{*}<\ln \mu_{j}-\beta^{\prime} X_{i}\right) \\
& =F\left(\ln \mu_{j}-\beta^{\prime} X_{i}\right)-F\left(\ln \mu_{j-1}-\beta^{\prime} X_{1}\right),
\end{aligned}
$$

donde $F(\bullet)$ denota la función de distribución acumulada. Habitualmente, se supone que dicha función de distribución es simétrica. En particular, si se supone que dicha función de distribución es la normal estándar, el modelo estadístico resultante es el probit ordenado. Este modelo puede estimarse por máxima verosimilitud. Además del vector de parámetros de interés, el modelo proporciona estimaciones de los puntos de corte de cada intervalo. Como ejemplo de una aplicación empírica de un modelo de respuesta ordenada, podemos citar a Papke (1998), que estudia las preferencias en la cartera de activos de los fondos de pensiones individuales (de más renta fija a más acciones del mercado bursátil).

Dado que el modelo estadístico solamente explota la información discreta sobre el carácter ordinal de la variable dependiente, la escala de los parámetros es irrelevante (estando determinada por la normalización de la varianza). En todo caso, tanto el signo como los valores relativos de los parámetros sí son relevantes, pudiendo esti-

3 Nuestra modelización supone que los individuos declaran el límite superior de cada intervalo. Evidentemente, el resultado estadístico no cambia si interpretamos que el individuo declara el límite inferior. La única diferencia estriba en la interpretación de los resultados y en la construcción de las predicciones individuales del salario a partir de los resultados de la estimación. 
marse los efectos marginales a partir de las estimaciones de los parámetros. Al tratarse de un modelo de elección discreta, las predicciones del modelo son las probabilidades de que el salario esperado declarado por cada individuo corresponda a cada una de las seis categorías.

No obstante, dado que conocemos los intervalos que determinan cada una de las categorías ordinales, el modelo tiene también un significado cuantitativo. Siguiendo a Wooldridge (2002), para una observación cualquiera, y utilizando la información sobre los límites que determinan cada una de las categorías salariales (en euros), podemos calcular la predicción del salario declarado como

$$
\begin{aligned}
W_{i}^{*} & =450 \operatorname{Pr}\left(d_{i j}=1 \mid X_{i}\right)+901 \operatorname{Pr}\left(d_{i j}=2 \mid X_{i}\right)+1803 \operatorname{Pr}\left(d_{i j}=3 \mid X_{i}\right) \\
& +3606 \operatorname{Pr}\left(d_{i j}=4 \mid X_{i}\right)+5409 \operatorname{Pr}\left(d_{i j}=5 \mid X_{i}\right)+1.33 \times 5409 \operatorname{Pr}\left(d_{i j}=1 \mid 6 X_{i}\right),
\end{aligned}
$$

donde para la última categoría, que no está acotada superiormente (más de 5409 euros), hemos aplicado el supuesto de que el salario sigue una distribución gamma con objeto de poder imputar el valor medio del salario para la categoría superior como 1.33 veces el límite inferior del intervalo. Esto nos permite comparar los salarios esperados y los salarios sombra predichos con los salarios medios obtenidos a partir de la Encuesta de Estructura Salarial para cada grupo.

\section{La valoración de la enseñanza superior: dos estudios complementarios}

Con la información sobre la valoración subjetiva que de la enseñanza superior tienen los estudiantes podemos plantear, entre otras, dos cuestiones de interés. En concreto, podemos ver cómo las características personales y familiares, así como el nivel de estudios de los individuos, condicionan su salario esperado. En segundo lugar, podemos ver cómo esos mismos factores afectan al salario sombra, es decir, a los ingresos salariales mínimos por los que estarían dispuestos a abandonar sus estudios superiores con un contrato indefinido.

Respecto al salario esperado, conviene recordar que la medida de que disponemos representa una valoración subjetiva. Este hecho complica la interpretación de los efectos totales de los factores en que se condiciona. Por un lado, dichos efectos recogen la influencia efectiva de tales factores en el salario potencial de los estudiantes. Por otro lado, los valores de estas variables capturan diferencias en la calidad de la información en la que cada individuo basa sus expectativas salariales.

En relación al salario sombra, podemos analizar en qué medida las características personales de los estudiantes universitarios, y otros factores tales como los estudios elegidos, afectan el nivel de renta por el que estarían dispuestos a abandonar sus estudios superiores. Este ejercicio tiene interés desde un doble punto de vista. Por un lado, permite observar cuál es la renta permanente que los estudiantes confían en ob- 
tener a la finalización de sus estudios y cómo influye en ella la elección de sus estudios. Por otro lado, conociendo las expectativas de los estudiantes sobre el nivel de renta permanente $-\mathrm{y}$ el salario esperado - al final de sus estudios es posible aseverar cuánto de realista es la estimación que los estudiantes están realizando sobre sus ingresos futuros.

A continuación, presentamos los resultados acerca de ambas cuestiones utilizando los resultados de encuestas realizadas en el ámbito geográfico de la Comunidad de Madrid en los años 2004 y 2005. Estos resultados conforman nuestra aproximación a la valoración económica de la enseñanza superior utilizando datos de la Comunidad de Madrid.

\subsection{El salario esperado y la enseñanza universitaria}

En esta sección estudiamos de qué forma las características personales y familiares y el nivel de estudios de los estudiantes condicionan las expectativas salariales después de finalizar sus estudios universitarios. Los resultados de las estimaciones del modelo probit ordenado para las submuestras de primer y segundo curso, tercer curso y cuarto y quinto curso se presentan en la Tabla 5. El ajuste del modelo, de acuerdo con el pseudo- $\mathrm{R}^{2}$ y con el contraste de Wald, es razonablemente bueno para las submuestras de cursos iniciales e intermedios, pero relativamente pobre para la submuestra de últimos cursos de carrera.

En esta especificación, la variable dicotómica relativa a la titulación universitaria del padre captura el efecto del entorno familiar. El hecho de que el padre tenga titulación universitaria afecta positivamente a la valoración subjetiva del individuo para los estudiantes que se hallan en cursos iniciales e intermedios de la carrera. Este efecto se torna negativo para los estudiantes que se encuentran en los últimos cursos de carrera, si bien no es estadísticamente significativo.

Si comparamos la media de los salarios esperados de nuestra muestra con los salarios efectivos obtenidos en la Tabla 3 para trabajadores de 20 a 29 años con educación superior en la Comunidad de Madrid, encontramos que las expectativas de salarios exceden en promedio al salario medio efectivo. Ello quiere decir que los estudiantes universitarios tienden a sobreestimar el salario potencial. En dicha sobreestimación, las características en que condicionamos afectan a la calidad de la información utilizada en el cómputo del salario esperado, y por tanto, al grado de sobreestimación. El problema radica en que estas características pueden recoger los efectos contrapuestos de cómo afecta cada característica concreta al salario potencial frente a cómo afecta dicha característica a la calidad en el cómputo de la expectativa salarial.

En el caso del efecto sobre el salario esperado de los estudios universitarios del padre, cabe esperar un efecto positivo sobre el salario potencial. Al mismo tiempo, cabe esperar que la calidad de la información sea superior en promedio para los estudiantes cuyo padre tiene estudios universitarios. En consecuencia, los individuos 
TABLA 5

MODELO DEL SALARIO ESPERADO. PROBIT ORDENADO

\begin{tabular}{|l|c|c|c|}
\hline & $\begin{array}{c}\mathbf{1}^{\text {er }} \mathbf{y} \mathbf{~ 2}^{\mathbf{o}} \\
\text { curso }\end{array}$ & $\begin{array}{c}\mathbf{3 .}^{\text {er }} \\
\text { curso }\end{array}$ & $\begin{array}{c}\mathbf{4 .}^{\mathbf{o}} \mathbf{y} \mathbf{5}^{\mathbf{o}} \\
\text { curso }\end{array}$ \\
\hline y2004 & $-0,5366$ & 0,1768 & $-0,0408$ \\
Selectividad & $(0,1122)$ & $(0,1402)$ & $(0,1664)$ \\
Mujer & 0,0051 & $-0,0008$ & 0,0069 \\
& $(0,0045)$ & $(0,0059)$ & $(0,0075)$ \\
Padre universitario & $-0,3028$ & 0,0076 & $-0,0431$ \\
& $(0,0777)$ & $(0,1050)$ & $(0,1271)$ \\
Repetidor & 0,2880 & 0,4953 & $-0,2015$ \\
& $(0,1140)$ & $(0,1629)$ & $(0,1497)$ \\
Ciencias & 0,1582 & 0,3815 & 0,2394 \\
& $(0,0804)$ & $(0,1090)$ & $(0,1350)$ \\
Busca & 0,0241 & 0,0780 & $-0,0812$ \\
Beca & $(0,0842)$ & $(0,1040)$ & $(0,1378)$ \\
& $-0,0431$ & 0,3030 & 0,0728 \\
Declara & $(0,0897)$ & $(0,1159)$ & $(0,1366)$ \\
& $-0,2019$ & $-0,1176$ & $-0,3661$ \\
& $(0,1078)$ & $(0,1336)$ & $(0,1671)$ \\
No. de obs. & $-0,0056$ & 0,0148 & 0,1821 \\
log-verosimilitud & $(0,1108)$ & $(0,1393)$ & $(0,1429)$ \\
Pseudo-R2 & 823 & 523 & 324 \\
Contraste de Wald & $-938,0$ & $-490,5$ & $-369,7$ \\
& 0,049 & 0,029 & 0,017 \\
& $95,3(0,0)$ & $29,1(2,9)$ & $12,7(17,5)$ \\
\hline
\end{tabular}

Fuente: Los Jóvenes ante la Universidad, 2004 y 2005.

Notas: En las estimaciones se ha incluido también una variable binaria y2004 que recoge el año (2004 ó 2005) en que se realizó la encuesta.

Errores estándar entre paréntesis debajo de cada coeficiente.

El Contraste de Wald evalúa la hipótesis nula de que los coeficientes de todas las variables en que se condiciona son iguales a cero. Se distribuye como una $\chi^{2}$ con 9 grados de libertad. El $p$-valor asociado (en tanto por ciento) se incluye entre paréntesis.

cuyo padre tiene titulación universitaria tenderán a sobreestimar menos el salario esperado respecto al salario efectivo. Este efecto se refuerza para los estudiantes en últimos cursos de carrera, en el que el efecto de la calidad de la información parece dominar.

El hecho de que el estudiante sea repetidor afecta positivamente al salario esperado. Por el contrario, el hecho de disfrutar de beca (aspecto positivamente relacionado con la calidad del estudiante) afecta negativamente al salario esperado. En este caso, parece claro que el aspecto informacional determina el signo del coeficiente. Sin 
embargo, estas variables no son significativas en todas las submuestras. La nota de selectividad, una vez que se controla por otras características, no tiene un efecto significativo en el cómputo del salario esperado. El resto de las variables incluidas no presentan efectos significativos.

A partir de los resultados obtenidos, podemos predecir las probabilidades respectivas de que el salario esperado corresponda a cada una de las seis categorías de la encuesta para a continuación predecir los salarios esperados individuales utilizando la información sobre los salarios en euros correspondientes a cada una de las categorías. Dicha información se presenta en la Tabla 6.

Los resultados de la Tabla 6 muestran que los estudiantes de cursos iniciales de la carrera tienen en promedio unas expectativas salariales muy superiores que las de los estudiantes de los cursos siguientes. Además, no se encuentran diferencias reseñables entre los salarios medios esperados de los estudiantes de tercer curso respecto a los de cuarto y quinto cursos. Comparando los salarios medios estimados con los salarios realmente observados para el colectivo comparable de titulados entre 20 y 29 años que están trabajando en la Comunidad de Madrid, encontramos que los estudiantes varones de primer y segundo curso esperan en promedio un salario mayor del salario medio observado. En el caso de las mujeres, esta diferen-

\section{TABLA 6}

\section{SALARIOS MEDIOS ESPERADOS POR LOS ESTUDIANTES UNIVERSITARIOS EN LA COMUNIDAD DE MADRID}

\begin{tabular}{|c|c|c|c|}
\hline & $1 .^{\mathrm{er}}$ y $2 .^{\circ}$ curso & 3. ${ }^{\text {er }}$ curso & $4 .^{\circ}$ y $5 .^{\circ}$ curso \\
\hline $\begin{array}{l}\text { Varones } \\
\text { Mujeres }\end{array}$ & $\begin{array}{l}2.720,20 \\
2.354,58\end{array}$ & $\begin{array}{l}2.176,20 \\
2.168,55\end{array}$ & $\begin{array}{l}2.179,56 \\
2.192,72\end{array}$ \\
\hline \multicolumn{4}{|c|}{$\begin{array}{l}\text { Diferencia porcentual entre las expectativas del salario medio de los estudiantes } \\
\text { y los salarios para licenciados realmente observados en España condicionado } \\
\qquad \begin{array}{l}\text { a encontrar un trabajo }\end{array}\end{array}$} \\
\hline & $1 .^{\mathrm{er}}$ y $2 .^{\circ}$ curso & 3. ${ }^{\text {er }}$ curso & $4 .^{\circ}$ y $5 .^{\circ}$ curso \\
\hline $\begin{array}{l}\text { Varones } \\
\text { Mujeres }\end{array}$ & $\begin{array}{l}24,90 \\
27,75\end{array}$ & $\begin{array}{r}-0,08 \\
17,65\end{array}$ & $\begin{array}{r}0,07 \\
18,96\end{array}$ \\
\hline \multicolumn{4}{|c|}{$\begin{array}{l}\text { Diferencia porcentual entre las expectativas del salario medio de los estudiantes y los salarios } \\
\text { para licenciados realmente observados en Madrid condicionado a encontrar trabajo }\end{array}$} \\
\hline & $1 .^{\mathrm{er}}$ y $2 .^{\circ}$ curso & 3. ${ }^{\text {er }}$ curso & $4 .^{\circ}$ y $5 .^{\circ}$ curso \\
\hline $\begin{array}{l}\text { Varones } \\
\text { Mujeres }\end{array}$ & $\begin{array}{r}8,60 \\
17,61\end{array}$ & $\begin{array}{r}-13,12 \\
8,32\end{array}$ & $\begin{array}{r}-12,99 \\
9,53\end{array}$ \\
\hline
\end{tabular}


cia positiva entre salario esperado medio y salario medio observado se produce para todos los cursos de carrera, si bien es mayor en términos porcentuales para primer y segundo curso. Asimismo, la sobreestimación de las expectativas salariales respecto al salario medio observado es más pronunciada en el caso de las mujeres. Mientras que sus expectativas salariales (salvo en primer y segundo curso) son bastante parecidas a las de los varones, las diferencias en los salarios observados por sexos son muy acusadas.

La sobreestimación es probablemente aún mayor si se tiene en cuenta que los colectivos de referencia para calcular los salarios esperados y salarios observados son diferentes. En el caso del salario medio observado, estamos condicionando en la submuestra de individuos que han decidido trabajar y han encontrado trabajo. Por el contrario, la muestra utilizada para calcular el salario medio esperado incluye tanto a individuos que se incorporarán a la población activa como a los que no, y de éstos, tanto a los que encontrarán trabajo nada más acabar sus estudios como a los que no. Por último, dado que un porcentaje de los estudiantes, usualmente los peores, abandonan sus estudios universitarios antes de la obtención de un título, es posible que la aparente mejora en la formación de expectativas refleje una selección muestral de individuos mejor informados en los dos últimos años de carrera.

\subsection{El salario sombra y la enseñanza universitaria}

En esta sección analizamos en qué medida las características personales de los estudiantes universitarios, y otros factores tales como los estudios elegidos, afectan el nivel de renta por el que estarían dispuestos a abandonar sus estudios superiores.

Los resultados de las estimaciones del modelo probit ordenado para las submuestras de primer y segundo curso, tercer curso y cuarto y quinto curso se presentan en la Tabla 7. El ajuste del modelo, de acuerdo con el pseudo- $\mathrm{R}^{2}$ y con el contraste de Wald, es razonablemente bueno para todas las submuestras, si bien empeora sustancialmente para la submuestra de últimos cursos de carrera, para la que el tamaño muestral es sustancialmente menor.

Los resultados para estudiantes de cursos iniciales o intermedios, que describimos a continuación, son muy similares y describen efectos que son coherentes con los resultados que cabría esperar. Entre las características académicas, la nota de selectividad y el hecho de ser repetidor, en tanto que medidas de la capacidad potencial del individuo, tienen efectos positivo y negativo, respectivamente, en el salario sombra. No obstante, mientras que el hecho de ser repetidor muestra un efecto muy significativo, la nota de selectividad sólo es marginalmente significativa en el caso de estudiantes de tercer curso. Las mujeres parecen tener en promedio un salario sombra menor, si bien el diferencial no es estadísticamente significativo. También resalta el hecho de que aquéllos que cursaron bachillerato de ciencias presentan en promedio un menor salario sombra, mientras que los estudiantes que están buscando empleo presentan en 
promedio un mayor salario sombra. Por último, el hecho de conocer la situación económica del hogar (a través de la variable Declara) o de que el padre tenga titulación universitaria aumenta su salario sombra.

En el caso de los estudiantes de últimos cursos, los resultados son menos precisos, con pocas variables significativas. En concreto, las características asociadas al rendimiento académico (nota de selectividad, ser repetidor, si cursó bachillerato de ciencias) son claramente no significativas. Las variables de mayor relevancia estadística en la determinación del salario sombra para los estudiantes de últimos cursos son el hecho de ser mujer, que tiene un efecto negativo muy marcado, si está buscando tra-

\section{TABLA 7}

MODELO DEL SALARIO SOMBRA. PROBIT ORDENADO

\begin{tabular}{|l|c|c|c|}
\hline & $\begin{array}{c}\mathbf{1}^{\text {er }} \mathbf{y ~ 2 .} \\
\text { curso }\end{array}$ & $\begin{array}{c}\mathbf{3}^{\text {er }} \\
\text { curso }\end{array}$ & $\begin{array}{c}\mathbf{4 .}^{\mathbf{o}} \mathbf{y} \mathbf{5}^{\mathbf{0}} \\
\text { curso }\end{array}$ \\
\hline y2004 & $-0,8331$ & $-0,4115$ & $-0,0755$ \\
Selectividad & $(0,1060)$ & $(0,1421)$ & $(0,1726)$ \\
Mujer & 0,0078 & 0,0109 & $-0,0045$ \\
& $(0,0043)$ & $(0,0059)$ & $(0,0080)$ \\
Padre universitario & $-0,0230$ & $-0,0198$ & $-0,2520$ \\
& $(0,0783)$ & $(0,0993)$ & $(0,1301)$ \\
Repetidor & 0,4850 & 0,6359 & 0,1827 \\
& $(0,1080)$ & $(0,1632)$ & $(0,1547)$ \\
Ciencias & $-0,4369$ & $-0,5196$ & $-0,1306$ \\
& $(0,0871)$ & $(0,1037)$ & $(0,1364)$ \\
Busca & $-0,1418$ & $-0,2233$ & 0,0418 \\
& $(0,0836)$ & $(0,1000)$ & $(0,1405)$ \\
Beca & 0,1567 & 0,6068 & 0,2619 \\
& $(0,0877)$ & $(0,1134)$ & $(0,1423)$ \\
Declara & $-0,4006$ & $-0,3197$ & 0,0982 \\
& $(0,1124)$ & $(0,1307)$ & $(0,1716)$ \\
\hline No. de obs. & 0,5147 & 0,7222 & 0,5099 \\
log-verosimilitud & $(0,1020)$ & $(0,1386)$ & $(0,1551)$ \\
Pseudo-R2 & 767 & 504 & 287 \\
Contraste de Wald & $-1.089,5$ & $-704,7$ & $-439,3$ \\
& 0,130 & 0,127 & 0,137 \\
& $324,6(0,0)$ & $205,6(0,0)$ & $29,2(0,1)$ \\
\hline
\end{tabular}

Fuente: Los Jóvenes ante la Universidad, 2004 y 2005.

En las estimaciones se ha incluido también una variable binaria y2004 que recoge el año (2004 ó 2005) en que se realizó la encuesta.

Errores estándar entre paréntesis debajo de cada coeficiente.

El Contraste de Wald evalúa la hipótesis nula de que los coeficientes de todas las variables en que se condiciona son iguales a cero. Se distribuye como una $\chi^{2}$ con 9 grados de libertad. El p-valor asociado (en tanto por ciento) se incluye entre paréntesis. 
bajo, que afecta (al igual que para cursos inferiores) de forma positiva, y si conoce la situación económica del hogar, con un efecto también positivo.

A partir de estos resultados, análogamente al modelo del salario esperado, podemos predecir los salarios sombra individuales y compararlos con los salarios medios observados. Esta comparación, que presentamos en la Tabla 8, nos proporciona el valor sombra de la educación universitaria para cada individuo de la muestra.

La Tabla 8 permite destacar varios aspectos. En primer lugar, los salarios sombra medios son, en esencia, mayores o iguales para las mujeres que para los hombres. Si se compara con los salarios observados, el salario sombra relativo al salario observado es mucho mayor en el caso de las mujeres. Este hecho refleja la menor tasa de participación y la mayor tasa de desempleo de las mujeres, incluso entre los titulados universitarios. Además, de acuerdo con Alba-Ramírez y San Segundo (1995), mientras que el rendimiento económico de la educación primaria y secundaria es menor para las mujeres que para los hombres, ocurre lo contrario en el caso de la educación universitaria. Este mayor rendimiento relativo de la educación universitaria para las mujeres sugiere que la inversión en educación universitaria es más atractiva para éstas que para los hombres. Y es asimismo consistente con el hecho de que la matrícula en la universidad española está dominada por las mujeres desde 1986.

\section{TABLA 8}

SALARIOS SOMBRA MEDIOS DE LOS ESTUDIANTES UNIVERSITARIOS EN LA COMUNIDAD DE MADRID

\begin{tabular}{|c|c|c|c|}
\hline & $10^{\mathrm{er}}$ y $20^{\circ}$ curso & $3{ }^{\text {er }}$ curso & $4 .^{o}$ y $5 .^{o}$ curso \\
\hline $\begin{array}{l}\text { Varones } \\
\text { Mujeres }\end{array}$ & $\begin{array}{l}3.619,576 \\
3.836,298\end{array}$ & $\begin{array}{l}3.250,900 \\
3.546,939\end{array}$ & $\begin{array}{l}4.097,615 \\
3.925,293\end{array}$ \\
\hline \multicolumn{4}{|c|}{$\begin{array}{l}\text { Diferencia porcentual entre los salarios sombra medios de los estudiantes } \\
\text { y los salarios para licenciados realmente observados en España condicional } \\
\text { a encontrar un trabajo }\end{array}$} \\
\hline & $10^{\mathrm{er}}$ y $20^{\circ}$ curso & $3 .^{\text {er }}$ curso & $4 .^{\circ}$ y $5 .^{\circ}$ curso \\
\hline $\begin{array}{l}\text { Varones } \\
\text { Mujeres }\end{array}$ & $\begin{array}{r}66,19 \\
108,13\end{array}$ & $\begin{array}{l}49,27 \\
92,44\end{array}$ & $\begin{array}{r}88,14 \\
112,96\end{array}$ \\
\hline \multicolumn{4}{|c|}{$\begin{array}{l}\text { Diferencia porcentual entre las expectativas del salario medio de los estudiantes y los salarios } \\
\text { para licenciados realmente observados en Madrid condicionado a encontrar trabajo }\end{array}$} \\
\hline & $10^{\mathrm{er}}$ y $20^{\circ}$ curso & $3 .^{\text {er }}$ curso & $4 .^{\circ}$ y $5 .^{\circ}$ curso \\
\hline $\begin{array}{l}\text { Varones } \\
\text { Mujeres }\end{array}$ & $\begin{array}{l}33,06 \\
62,93\end{array}$ & $\begin{array}{l}49,38 \\
63,56\end{array}$ & $\begin{array}{l}88,00 \\
79,01\end{array}$ \\
\hline
\end{tabular}


En segundo lugar, el menor salario sombra para ambos sexos se da para los estudiantes que se encuentran en tercer año (curso intermedio), alcanzado su mayor valor para los últimos cursos de carrera (cuando el coste de oportunidad de abandonar es más alto).

\section{Conclusiones}

Este trabajo intenta aportar respuestas al problema de valoración económica de la educación universitaria a través de las valoraciones subjetivas de los estudiantes universitarios sobre sus propios estudios. Para ello, utilizamos una base de datos microeconómica para los años 2004 y 2005 que, además de características académicas, personales y familiares, recoge las declaraciones de los individuos acerca de sus salarios esperados a la finalización de los estudios universitarios y los salarios sombra (mínimo a partir del cual abandonarían sus estudios para trabajar con un contrato indefinido percibiendo dicho salario). El hecho de que los salarios declarados estén codificados de forma discreta en seis intervalos imposibilita realizar un análisis de regresión lineal. El modelo estadístico que permite un tratamiento apropiado de los datos observados consiste en un modelo de respuesta ordenada, que consiste en un modelo multinomial que tiene en cuenta el carácter discreto pero ordinal de la variable dependiente. Dado que el curso en que cada individuo se encuentra puede afectar a la valoración subjetiva de los estudios universitarios al existir diferencias en el horizonte de terminación de los estudios, los modelos para salarios esperados y salarios sombra se estiman para tres submuestras correspondientes al momento en que se hallan cursando sus estudios: primer y segundo curso, tercer curso, cuarto y quinto curso. Los principales resultados se resumen a continuación.

En lo que respecta a los salarios esperados, la interpretación de los efectos totales de los factores en que se condiciona combina dos efectos que pueden ser contrapuestos, como son la influencia efectiva de tales factores en el salario potencial, por un lado, y el reflejo de la calidad de la información en la que cada individuo basa sus expectativas salariales, por otro. Asimismo, existen diferencias notables en los efectos de los factores en que se condiciona dependiendo del curso en que el estudiante se encuentra. En concreto, variables del entorno familiar como tener un padre con estudios universitarios afectan positivamente a la valoración subjetiva del salario en cursos iniciales e intermedios, pero su efecto cambia de signo para los estudiantes en sus últimos años de carrera. En general, el efecto de las variables sobre la calidad de la información en que basan sus expectativas parece dominar, particularmente en el caso de los estudiantes próximos a finalizar sus estudios. Asimismo, la comparación de los salarios esperados predichos utilizando el modelo estimado y los salarios observados para un colectivo de trabajadores comparable muestra que las expectativas se hacen más realistas para cursos más avanzados de la carrera, lo que refleja que el conjunto de información en que el es- 
tudiante basa el cálculo mejora a medida que se acerca la finalización de los estudios. Además, encontramos una sobreestimación de las expectativas salariales por parte de los estudiantes de ambos sexos en primeros cursos de carrera, así como para las mujeres en cualquier curso de carrera. En este último resultado se combina el hecho de que las expectativas salariales son similares para ambos sexos con que los salarios efectivamente observados son sustancialmente menores para las mujeres.

En cuanto a los determinantes del salario sombra, los resultados son coherentes con lo que cabría esperar. En concreto, el rendimiento académico y el hecho de que el padre tenga estudios universitarios afecta positivamente a dicho salario sombra. Asimismo, para los últimos cursos de carrera, el hecho de ser mujer tiene un marcado efecto negativo, mientras que estar buscando empleo afecta positivamente. Las predicciones de los salarios sombra obtenidas a partir de las estimaciones son también coherentes económicamente. En concreto, el salario sombra más alto corresponde a estudiantes en sus últimos años de carrera (cuando el coste de oportunidad de abandonar es más alto). Otro resultado interesante es que los salarios sombra son en media mayores o iguales para las mujeres que para los hombres, pese a que los salarios observados son sistemáticamente más bajos para las mujeres. Esta evidencia es consistente con el hecho de que las mujeres obtienen un mayor rendimiento económico relativo de la educación universitaria que los hombres, así como el predominio de las mujeres entre los jóvenes matriculados en la universidad en los últimos veinte años. Por último, el menor salario sombra tiene lugar para estudiantes en el curso intermedio de ambos sexos, resultado para el que no disponemos de una explicación económica clara.

El trabajo presenta, no obstante, algunas limitaciones de cierta importancia. Estas se refieren a la existencia de sesgo de selección, a la información explotada en la estimación del modelo, y a ciertos problemas de identificación relativos a la duración de las carreras cursadas.

En lo que respecta al sesgo de selección, un porcentaje sustancial de los encuestados no contestaron a las preguntas sobre salarios esperados y salarios sombra (alrededor del 14 y del 20 por ciento respectivamente). Sería por tanto interesante ampliar el modelo econométrico para controlar por la no respuesta y estudiar si las probabilidades de responder a estas preguntas están influidas sistemáticamente por factores que a su vez condicionan las respuestas. Otro problema de selección muestral, ya mencionado anteriormente, tiene que ver con el hecho de que la muestra de estudiantes después del primer curso de carrera está sujeta a desgaste muestral no aleatorio derivada de la pérdida de observaciones de aquellos individuos que van abandonando (voluntaria o involuntariamente) la carrera universitaria. Sin embargo, este último problema es difícilmente tratable, al no disponer de información individual sobre aquellos estudiantes que abandonan la universidad.

En cuanto al modelo estadístico, aunque nuestro procedimiento no precisa conocer los umbrales de cada categoría en las respuestas sobre salarios esperados y sombra (que son estimados como parámetros incidentales), dichos umbrales son 
en nuestro caso conocidos. La posibilidad de explotar la información sobre los umbrales permitiría mejorar la precisión de las estimaciones y, sobre todo, la interpretación de éstas. Ello podría hacerse con un cierto esfuerzo de programación, formulando un modelo que incorpore la información sobre los umbrales conocidos como si se tratase de un modelo con múltiples puntos conocidos de censura. La ventaja de esta alternativa es que la escala de los parámetros estimados no sería arbitraria, de manera que dichos parámetros proporcionarían directamente los efectos marginales.

Debido a la información disponible sin necesidad de edición en la encuesta, la especificación de las variables elegida no ha permitido diferenciar entre titulaciones de ciclo corto (diplomaturas) y de ciclo largo (licenciaturas). Asimismo, tampoco es posible distinguir titulaciones técnicas. Sin embargo, algunas de las cuestiones que han quedado abiertas en nuestro trabajo (en particular, la estructura en forma de U de los salarios sombra en relación al curso académico) podrían ser resueltas si se pudiera controlar por las características de la titulación cursada.

\section{Referencias bibliográficas}

[1] ALBA-RAMÍREZ, A. y SAN SEGUNDO, M.J. (1995): «The Returns to Education in Spain», Economics of Education Review 14(2), 155-66.

[2] BLINDER, A.S. y WEISS, Y. (1976): «Human Capital and Labor Supply: A synthesis>, Journal of Political Economy, 84, 449-72.

[3] CIS (1991): Los Jóvenes Ante el Sistema Educativo. Diseño y redacción, Margarita Latiesa, Centro de Investigaciones Sociológicas, Estudios y Encuestas; 25.

[4] FINA, L. GARCÍA SERRANO C., MAÑÉ, F. y TOHARIA, L. (2000): «Cambio ocupacional y necesidades educativas de la economía española», en F. Sáez (coord.): Formación y empleo. Madrid, Fundación Argentaria, 2000, 47-154.

[5] HALEY, W.J. (1976): «Estimation of the Earnings Profile from Optimal Human Capital Accumulation», Econometrica, 44, 1223-38.

[6] PAPKE, L.E. (1998): «How Are Participants Directing Their Participant-Directed Individual Account Pension Plans? 》, American Economic Review, 88, 212-216.

[7] PETRONGOLO, B. y SAN SEGUNDO, M.J. (2002): «Staying-on at school at sixteen. The impact of labor market conditions in Spain〉, Economics of Education Review.

[8] ROSEN, S. (1976): «A Theory of Life Earnings», Journal of Political Economy, 54, S45-67.

[9] SAN SEGUNDO, M.J. (1997): «Educación e Ingresos en el Mercado de Trabajo Español〉, Cuadernos Económicos de Información Comercial Española, número 63, 105-123.

[10] SAN SEGUNDO, M.J. (2002): «El Sistema Universitario Español en una Perspectiva Internacional», en Hernández, J. (comp.): La universidad española en cifras, CRUE, Madrid. 
[11] SALAS, M. y MARTÍN-COBOS PUEBLA, M. (2006): «La Demanda de Educación Superior: Un Análisis Microeconómico con Datos de Corte Transversal». Revista de Educación, 339, 637-660.

[12] TROSTEL, P. (2004): «Returns to Scale in Producing Human Capital from Schooling». Oxford Economic Papers, 56, 461-484.

[13] WOOLDRIDGE, J.M. (2002): Econometric Analysis of Cross Section and Panel Data, MIT Press. Cambridge, Massachussets. 
